\title{
Biofilm formation in Campylobacter jejuni
}

Correspondence

B. W. Wren

brendan.wren@Ishtm.ac.uk

Received 19 July 2005

Revised 21 October 2005

Accepted 10 November 2005

\author{
G. W. P Joshua, C. Guthrie-Irons, A. V. Karlyshev and B. W. Wren \\ Department of Infectious and Tropical Diseases, London School of Hygiene and Tropical \\ Medicine, Keppel Street, London WC1E 7HT, UK
}

\begin{abstract}
The major gastrointestinal pathogen Campylobacter jejuni is shown to exist as three forms of monospecies biofilm in liquid culture. It attaches to a glass surface; forms an unattached aggregate (floc); and forms a pellicle at the liquid-gas interface. The three forms of biofilm resemble each other when examined by scanning electron microscopy. The biofilm mode of growth confers protection against environmental stress, the microaerobic bacteria in flocs surviving up to 24 days at ambient temperature and atmosphere compared to 12 days survival by planktonic bacteria. The wild-type strains C. jejuni 33106, 32799, 33084 and 31485 did not form flocs, and floc formation was reduced in strains mutant in a putative flagellar protein (FliS) and in a phosphate acetyltransferase (Cj0688). All other strains tested, including strains with mutations affecting capsular polysaccharide (kpsM), flagella (maf5), protein glycosylation ( $p g / H)$ and lipo-oligosaccharide (neuB1) formed flocs. Similarly, all strains tested formed a pellicle and attached to glass except the aflagellate mutant maf5; pellicle formation was reduced in fliS and cj0688 mutants. Different mechanisms, therefore, may control formation of different forms of biofilm. It is proposed that these poorly characterized forms of growth are important for the persistence of C. jejuni in the environment and may in part explain the high incidence of Campylobacter-associated food borne disease.
\end{abstract}

\section{INTRODUCTION}

Campylobacter jejuni, a Gram-negative, microaerophilic bacterium, is the most frequently isolated foodborne pathogen worldwide. The annual reported incidence in England and Wales is around 50000 , which is thought to be a gross underestimate (CPLS, 2000). C. jejuni is also associated with neuropathies such as Guillain-Barré and Miller-Fisher syndromes (Salloway et al., 1996), where it is postulated that there is molecular mimicry between $C$. jejuni lipooligosaccharide and human gangliosides, leading to an auto-immune response (Yuki, 1997). The primary source of human infection is thought to be the consumption or handling of contaminated chicken and therefore the survival of C. jejuni in poultry house water systems is particularly significant (Pearson et al., 1996; Zimmer et al., 2003).

There is a paradox in that although C. jejuni is extremely widespread, its microaerobic growth requirements mean that the organism does not multiply in the natural aerobic environment. Although not all 'natural environments' that C. jejuni inhabits may necessarily be aerobic, it is still difficult to explain the high incidence of infection, particularly as in contrast to several other gastrointestinal pathogens, $C$. jejuni is rarely transmitted between humans. It has been suggested that $C$. jejuni maintains itself in the environment

Abbreviations: CLSM, confocal laser scanning microscopy; EPM, extracellular polymeric matrix; SEM, scanning electron microscopy. by forming a biofilm (Buswell et al., 1998). Biofilms are commonly defined as matrix-enclosed bacterial populations adherent to each other and/or to surfaces or interfaces (Costerton et al., 1995). Aggregates of bacteria not attached to a surface are commonly termed flocs and have many of the characteristics of a biofilm (Hall-Stoodley et al., 2004). Pellicles are aggregates of bacterial cells that form at an airliquid interface (Friedman \& Kolter, 2004).

The biofilm mode of growth is widespread and is of economic importance in diverse ecological niches (Costerton et al., 1995). Monospecies biofilms are formed by many human pathogens such as Pseudomonas aeruginosa (Whiteley et al., 2001), Staphylococcus epidermidis (Mack et al., 2000), Salmonella enteritidis (Solano et al., 2002), Vibrio cholerae (Nesper et al., 2001), Streptococcus gordonii (Loo et al., 2000) and Burkholderia cepacia (Huber et al., 2001). In human infection, biofilms are often less susceptible to antibiotics (Nichols, 1991).

C. jejuni has been observed to autoagglutinate, in MuellerHinton broth, minimal essential medium (Golden \& Acheson, 2002) and phosphate-buffered saline (Misawa \& Blaser, 2000). However, it is unclear if autoagglutination represents a biofilm mode of growth in this species. C. jejuni has been found in preformed biofilms of other bacterial species (Trachoo et al., 2002; Keevil, 2003). Buswell et al. (1998) postulated that C. jejuni in autochthonous biofilms had enhanced survival. C. jejuni adherent to stainless steel 
coupons were assumed to have formed biofilms by Somers et al. (1994). However, to our knowledge, biofilm formation by $C$. jejuni has not hitherto been properly demonstrated. We therefore examined C. jejuni, grown in liquid culture, with and without shaking; and on glass or cellulose acetate surfaces by confocal laser scanning microscopy (CLSM) and by scanning electron microscopy (SEM) to determine whether it can use this form of growth. Additionally, selected C. jejuni mutants were tested for their ability to form biofilms to help identify likely surface determinants that may play a role in biofilm formation.

\section{METHODS}

Growth. All C. jejuni cultures were grown in a Variable Atmosphere Incubator (Don Whitley Scientific) under microaerobic conditions $\left(\mathrm{N}_{2}, 85 \% ; \mathrm{O}_{2}, 5 \% ; \mathrm{CO}_{2}, 10 \%\right)$ at $37^{\circ} \mathrm{C}$. For collection of bacterial aggregates, cultures were in Mueller-Hinton broth shaken at 50 r.p.m. on a mini orbital shaker (Stuart Scientific). Ten millilitres of medium was seeded with $100 \mu \mathrm{l}$ Mueller-Hinton broth containing a $10 \mu \mathrm{l}$ loop of $C$. jejuni taken from a Columbia blood agar plate containing $6 \cdot 8 \%(\mathrm{v} / \mathrm{v})$ horse blood, grown for 2 days, also under microaerobic conditions. Aggregate and planktonic bacteria were grown for 3 days in vented $50 \mathrm{ml}$ tissue flasks (Falcon).

For growth of pellicles and attached bacteria, cultures were in $10 \mathrm{ml}$ Brucella broth seeded with $C$. jejuni from Columbia blood plates to an $\mathrm{OD}_{600}$ of $0 \cdot 1-0 \cdot 2$ in glass test tubes incubated without shaking under microaerobic conditions (see above) at $37^{\circ} \mathrm{C}$ for 5 days.

Bacterial strains and mutants (see Table 1). C. jejuni strains NCTC 11168 (National Culture Type Collection, London, UK), its stable motile derivative $11168 \mathrm{H}$ (Karlyshev et al., 2000) and 81-176
(Black et al., 1988) have been described elsewhere. Clinical isolates were from an Infectious Intestinal Disease case control study (strains 31467, 33106, 32799, 33084 and 31485) and a C. jejuni outbreak in Wales in 2000 (strain 40671). These strains were obtained from the Campylobacter Reference Laboratory (Health Protection Agency, UK). Mutant strains were made in $11168 \mathrm{H}$ and included mutations in genes affecting capsular polysaccharide formation ( $k p s M$, encoding a capsule transport protein) (Karlyshev et al., 2000), flagella formation (maf5 is located in the flagellin glycosylation locus, and the maf5 mutant is aflagellate and non-motile) (Karlyshev et al., 2002), $\mathrm{N}$-linked protein glycosylation biosynthesis ( $p g l \mathrm{H}$, encoding a glycosyltransferase essential for the biosynthesis of the $\mathrm{N}$-linked protein glycosylation pathway) (Linton et al., 2002) and lipo-oligosaccharide biosynthesis (neuB1, encoding a sialic acid synthetase) (Linton et al., 2000). In order to complement the maf5 mutation in $11168 \mathrm{H}$, the maf5 gene was PCR amplified and cloned downstream from the $\mathrm{cam}^{\mathrm{r}}$ gene from the delivery vector pAV35 (van Vliet et al., 1998) under the control of a $\mathrm{cam}^{\mathrm{r}}$ promoter constitutively expressed in Campylobacter. Integration of the $\mathrm{cam}^{\mathrm{r}}$ maf5 gene fusion cassette into the $r r s$ (16S rRNA) $-r r l$ (23S rRNA) spacer region of one of the three rRNA gene clusters in the chromosome resulted in restoration of motility of the $11168 \mathrm{H}$ maf5 mutant (Karlyshev \& Wren, 2005). The complemented $11168 \mathrm{H}$ maf5 mutant was termed $11168 \mathrm{H}$ maf5$\left(\mathrm{maf} 5^{+}\right)$. Mutants fliS, cj0688 and cj0689 were generated via insertion of the $k \mathrm{r}^{\mathrm{r}}$ cassette from the delivery vector pJMK30 (van Vliet et al., 1998) into the respective pUC18 derivatives obtained from a random $2 \mathrm{~kb}$ library generated in the $C$. jejuni NCTC 11168 genome sequencing project (Parkhill et al., 2000). All wild-type strains were motile, as were all mutants except maf5 and fliS, which were aflagellate non-motile mutants. All strains and mutants used in the study had similar growth profiles in Mueller-Hinton liquid culture medium over a $48 \mathrm{~h}$ period (data not shown).

Microscopy. For CLSM (Zeiss HAL 100), bacteria on glass cover slips were mounted in PBS/glycerol $(1: 1, \mathrm{v} / \mathrm{v})$ and visualized with

Table 1. Strains of $C$. jejuni used and their ability to form aggregates, pellicles and attached biofilms and their relative hydrophobicity

\begin{tabular}{|lcccc|}
\hline Strain & Aggregate $^{*}$ & Attached $^{*}$ & Pellicle $^{\star}$ & Hydrophobicity $^{\dagger}$ \\
\hline NCTC 11168 & +++ & ++ & ++ & $0 \cdot 004 \pm 0 \cdot 002$ \\
$11168 \mathrm{H}$ & ++ & ++ & ++ & $0 \cdot 023 \pm 0 \cdot 005$ \\
$11168 \mathrm{H} \mathrm{kpsM}$ & +++ & +++ & +++ & $0 \cdot 004 \pm 0 \cdot 002$ \\
$11168 \mathrm{H}$ maf5 & ++ & - & - & $0 \cdot 875 \pm 0 \cdot 072$ \\
$11168 \mathrm{H} \mathrm{maf5}\left(\right.$ maf5 $\left.^{+}\right)$ & ++ & ++ & ++ & $0 \cdot 0008 \pm 0$ \\
$11168 \mathrm{H}$ pglH & ++ & ++ & ++ & $0 \cdot 001 \pm 0 \cdot 001$ \\
$11168 \mathrm{H} \mathrm{neuB1}$ & ++ & +++ & +++ & $0 \cdot 001 \pm 0$ \\
$11168 \mathrm{H} \mathrm{fliS}$ & + & - & + & $0 \cdot 333 \pm 0 \cdot 058$ \\
$11168 \mathrm{H} \mathrm{cj0688}$ & + & + & + & $0 \cdot 027 \pm 0 \cdot 008$ \\
$11168 \mathrm{H} \mathrm{cj0689}$ & ++ & ++ & ++ & $0 \cdot 013 \pm 0 \cdot 001$ \\
$81-176$ & ++ & ++ & ++ & Not tested \\
40671 & ++ & ++ & ++ & $0 \cdot 078 \pm 0 \cdot 027$ \\
31467 & ++ & ++ & ++ & $0 \cdot 031 \pm 0$ \\
33106 & - & ++ & ++ & $0 \cdot 046 \pm 0 \cdot 015$ \\
32799 & - & ++ & ++ & $0 \cdot 038 \pm 0 \cdot 004$ \\
33084 & - & ++ & ++ & $0 \cdot 038 \pm 0 \cdot 004$ \\
31845 & - & ++ & + & $0 \cdot 002 \pm 0$ \\
\hline
\end{tabular}

${ }^{\star}-$, absent; + , small (just visible); ++ , intermediate; +++ , extensive.

$\dagger$ Minimal concentration of ammonium sulphate for cell aggregation of $0 \cdot 005-0 \cdot 05,0 \cdot 05-0 \cdot 25$ and $0 \cdot 25-1 \cdot 0 \mathrm{M}$ represents strong, medium and weak hydrophobicity, respectively. 
SYTO 16 (Molecular Probes). For indirect immunofluorescence tests (IFATs), Penner 2 antiserum was used at 1:100 and visualized with Alexa 546 (Molecular Probes). For SEM, aggregates were transferred to cover slips with a wide-bore pipette; pellicles were transferred by dipping cover slips through the surface of standing cultures. Attached bacteria were obtained by breaking the glass test tube and processing bacteria in situ. Bacteria were fixed for $2 \mathrm{~h}$ in $3 \%$ glutaraldehyde made up in $0.2 \mathrm{M}$ sodium cacodylate buffer $\mathrm{pH} 7 \cdot 4$. The samples were then washed in the same buffer for 75 min prior to post-fixing in $1 \%$ osmium tetroxide in the $0.2 \mathrm{M}$ sodium cacodylate buffer for $105 \mathrm{~min}$. Subsequent washing in $0 \cdot 2 \mathrm{M}$ buffer for a further $25 \mathrm{~min}$ was followed by the storage at $4{ }^{\circ} \mathrm{C}$ in $0.2 \mathrm{M}$ sucrose in $0.2 \mathrm{M}$ sodium cacodylate overnight. The samples were then washed in two changes of MilliQ water over $145 \mathrm{~min}$ and dehydrated through a graduated series of ethanol (15-30 min per step; $30 \%, 50 \%, 70 \%$, $90 \%, 2 \times 100 \%)$. They were subsequently air-dried in a ventilated covered container and sputter-coated in an Edwards S150 sputter coater. The samples were examined on a JEOL JSM25III scanning electron microscope and photographed using Kodak 120 TP black and white film. Kodak D19 developer was used for developing the rolls of film and the images were printed on Agfa multi-grade paper using an Agfa Print Processor and Agfa chemistry.

Hydrophobicity assay. The relative hydrophobicity of cells was determined using the 'salting out' method of Misawa \& Blaser (2000). Hydrophobicity was assessed on the minimum concentration of ammonium sulphate permitting aggregation of cells using a serial doubling dilution of a $4 \mathrm{M}$ solution.

Survival assay. For comparison of survival of aggregate and planktonic bacteria, $10 \mathrm{ml}$ cultures in Mueller-Hinton liquid medium were grown at 50 r.p.m. for 3 days at $37^{\circ} \mathrm{C}$ and transferred to test tubes. Aggregates were allowed to settle under gravity for $10 \mathrm{~min}$, washed three times in broth and resuspended in $10 \mathrm{ml}$ fresh Mueller-Hinton liquid medium. Similarly, planktonic bacteria in the supernatant were centrifuged at 4000 r.p.m., washed three times in broth and resuspended in $10 \mathrm{ml}$ fresh Mueller-Hinton liquid medium. Cultures were then stored at ambient temperature and atmospheric conditions for the duration of the assay. At 3 day intervals viable bacterial counts were determined on Columbia blood-agar plates. Experiments were performed in triplicate.

\section{RESULTS}

In our initial studies we attempted to grow biofilms of the sequenced strain of $C$. jejuni (NCTC 11168) on glass cover slips or on cellulose acetate filters, either in tissue culture flasks, in 24-well tissue culture plates or in a modified Robbins device (Tyler Engineering) (Domingue et al., 1994). We found that in the modified Robbins device, no bacteria attached to either cellulose acetate filters or glass with a flow rate of $300 \mathrm{ml} \mathrm{h}^{-1}$, as used by Domingue et al. (1994) or with various flow rates down to $10 \mathrm{ml} \mathrm{h}^{-1}$. Nor did C. jejuni NCTC 11168 attach to polystyrene, as assessed by crystal violet staining, in tests to quantify biofilm formation (Nesper et al., 2001) (data not shown). Similarly, in liquid culture with normal shaking (80-100 r.p.m.) there was no growth of bacteria on the plastic of the tissue culture flask, or on the surface of glass cover slips or cellulose acetate filters, and bacteria in the medium were planktonic. However, with shaking at a lower speed (50 r.p.m.) we noted the formation of bacterial aggregates in culture. We also observed the formation of a pellicle at the liquid-gas interface of cultures grown without shaking after 3-6 days, with cells impinging on the walls of the test tubes at the liquid-gas interface strongly attached.

We therefore tested a variety of strains of $C$. jejuni for their ability to form aggregates and pellicles, and to attach to glass. These included several clinical isolates, NCTC 11168, its stable motile derivative $11168 \mathrm{H}$ and several $11168 \mathrm{H}$ isogenic mutants. Three strains contained mutations in genes $k p s M$, $n e u B 1$ and $p g l H$, affecting the biosynthesis of three surface glyco-conjugants on C. jejuni cells, namely capsular polysaccharide, lipo-oligosaccharide and $N$-linked glycoproteins respectively. Selection of these mutants was based on the observation that in other bacteria an extracellular matrix in the biofilms may contain polymers of carbohydratecontaining molecules (Danese et al., 2000; Mack et al., 2000; Nesper et al., 2001; Solano et al., 2002; Sherlock et al., 2005). The maf5 and fliS mutants are aflagellate and were chosen because of a possible role of flagella as an adhesin during an initial step of biofilm formation (Kirov et al., 2004; Watnick et al., 2001; Golden \& Acheson, 2002). cj0688 and cj0689 encode putative phosphate acetyltransferase and acetate kinase respectively. Since the latter two genes have recently been shown to be involved in the biofilm formation in Escherichia coli (Wolfe et al., 2003) we reasoned that $C$. jejuni homologues of these genes may also affect biofilm formation.
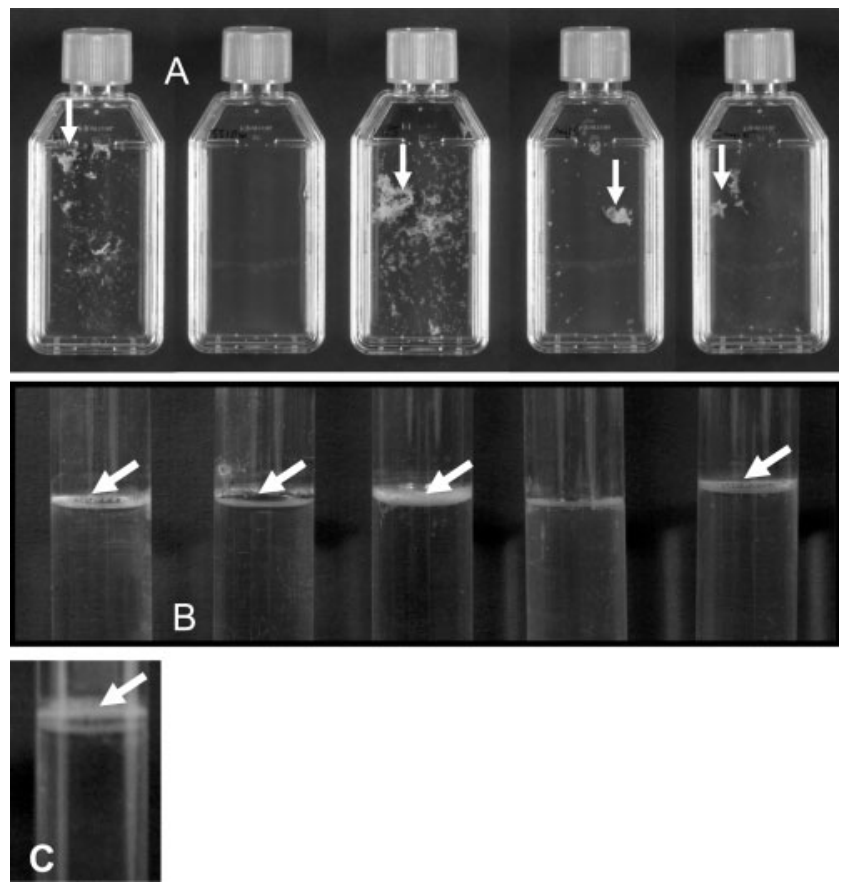

Fig. 1. Aggregates (A), pellicles (B) and attached (C) C. jejuni biofilms. Panels $A$ and $B$ show $11168 \mathrm{H}, 11168 \mathrm{H}$ fliS, $11168 \mathrm{H}$ kpsM, $11168 \mathrm{H}$ maf5 and $11168 \mathrm{H} \mathrm{maf5}\left(\mathrm{maf5}^{+}\right)$from left to right. Panel $\mathrm{C}$ shows $11168 \mathrm{H}$ standing culture after removal of medium. Aggregate (A) and attached biofilms (B, C) are arrowed. (Tissue culture flasks and test tubes, not to scale.) 
Excepting C. jejuni wild-type strains 33106, 32799, 33084 and 31485, all strains tested formed aggregates, including the isogenic mutants $p g l H, k p s M$, neuB1 and maf5. Aggregates in fliS and in cj0688 mutants were reduced. All wildtype strains also formed pellicles and attached to the surface of glass test tubes, including strains 33106, 32799, 33084 and 31485. The $\mathrm{pglH}$ mutant formed a pellicle and attached to glass in a similar way to wild-type strains; the $k p s M$ and neuB1 mutants formed a noticeably larger pellicle than all other strains. The maf5 mutant did not form a pellicle or attach to glass but this ability was restored in the complemented strains. Pellicle formation was also reduced in the fliS and cj0688 mutants. Fig. 1 shows examples of the various forms of biofilm. Note that attached bacteria were contiguous with the pellicle, and thus are not easily seen in intact standing cultures (Fig. 1B); Fig. 1C shows attached $11168 \mathrm{H}$ bacteria after removal of a portion of medium. The results are summarized in Table 1; all strains were tested
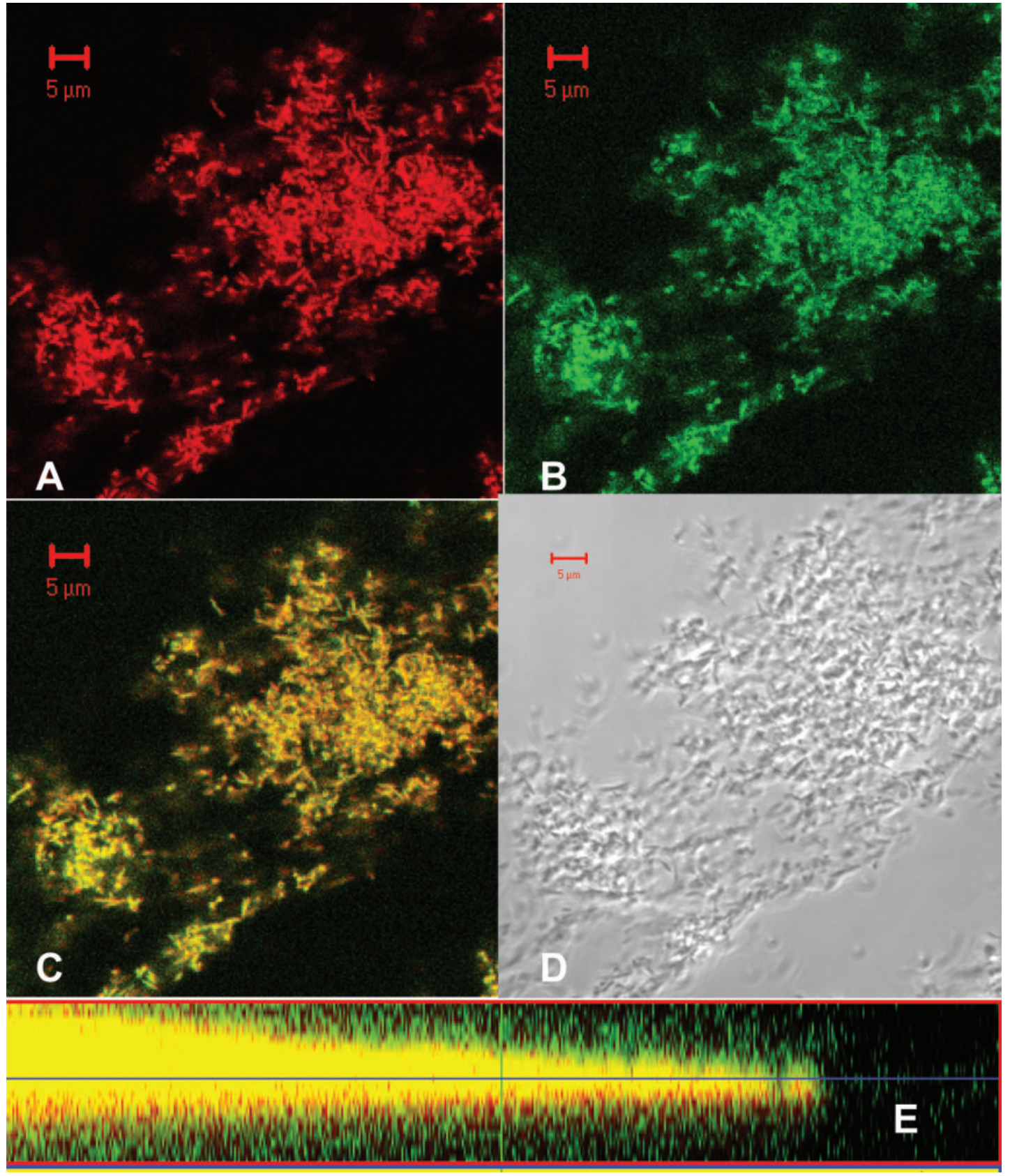

Fig. 2. CLSM of C. jejuni 11168H aggregate biofilm on glass coverslip. A, stained with Penner 2 antiserum visualized with Alexa 546; B, stained with SYTO 16; C, Penner 2 and SYTO 16 merged; D, phase-contrast. Bars, $5 \mu \mathrm{m}$. E, orthogonal projection from Z-stack of aggregate biofilm. 
in triplicate (mutant strains using three clonal isolates). Additionally, to provide information on the potential physical forces responsible for cell-cell interactions, Table 1 includes the relative hydrophobicity values for each strain and mutant strain studied.

We examined aggregates, pellicles and attached forms of bacteria in $11168 \mathrm{H}$ and selected mutants. Fig. 2 shows CLSM images of $C$. jejuni $11168 \mathrm{H}$ aggregates on glass cover slips. Under phase-contrast (Fig. 2D) a mass of bacteria can be seen as curved rods which were morphologically identifiable as $C$. jejuni. These were stained by the nucleic acid specific stain SYTO 16 (Fig. 2B) and Penner 2 antiserum, specific to $11168 \mathrm{H}$, (Fig. 2A, and shown merged in Fig. 2C). The three-dimensional nature of the bacterial mass, characteristic of a biofilm, is shown by orthogonal projection from a Z-stack (Fig. 2E). However, CLSM does not show connection of the bacteria by an extracellular polymeric matrix $(\mathrm{EPM})$, which is a defining characteristic of a biofilm. This is seen by SEM (Figs 3-5).

Fig. 3 shows the three forms of biofilm of C. jejuni $11168 \mathrm{H}$. Fig. 3A shows the bacterial aggregates grown in liquid culture. The extracellular material connecting bacteria appears flattened and extensive. Biofilm bacteria attached to glass (Fig. 3B) resemble the aggregate form, with a flattened and extensive EPM. In the pellicular biofilm (Fig. 3C), however, bacteria are similarly connected via extracellular material but this material is not extensive or flattened.
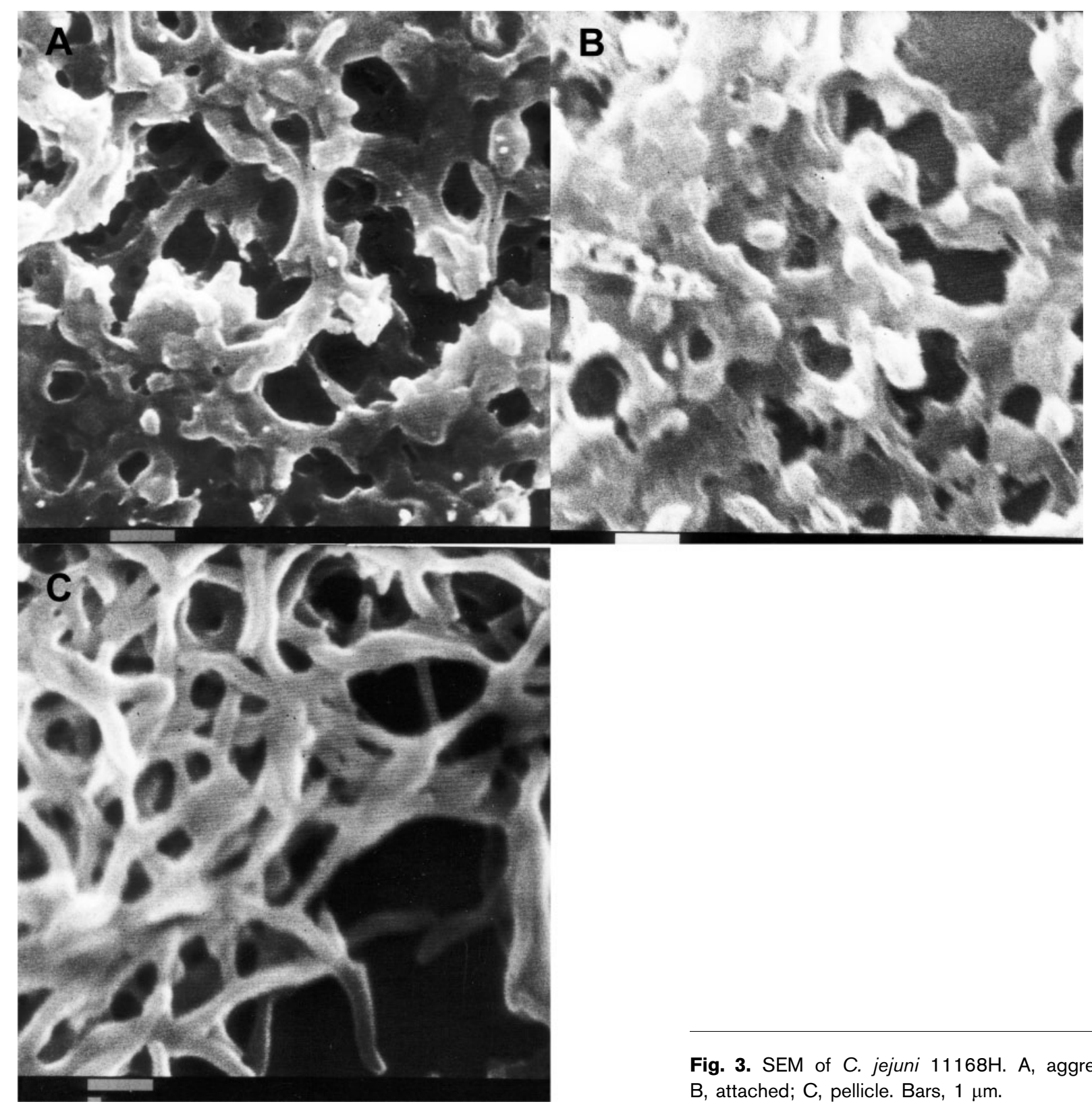

Fig. 3. SEM of C. jejuni $11168 \mathrm{H}$. A, aggregate; $\mathrm{B}$, attached; C, pellicle. Bars, $1 \mu \mathrm{m}$. 

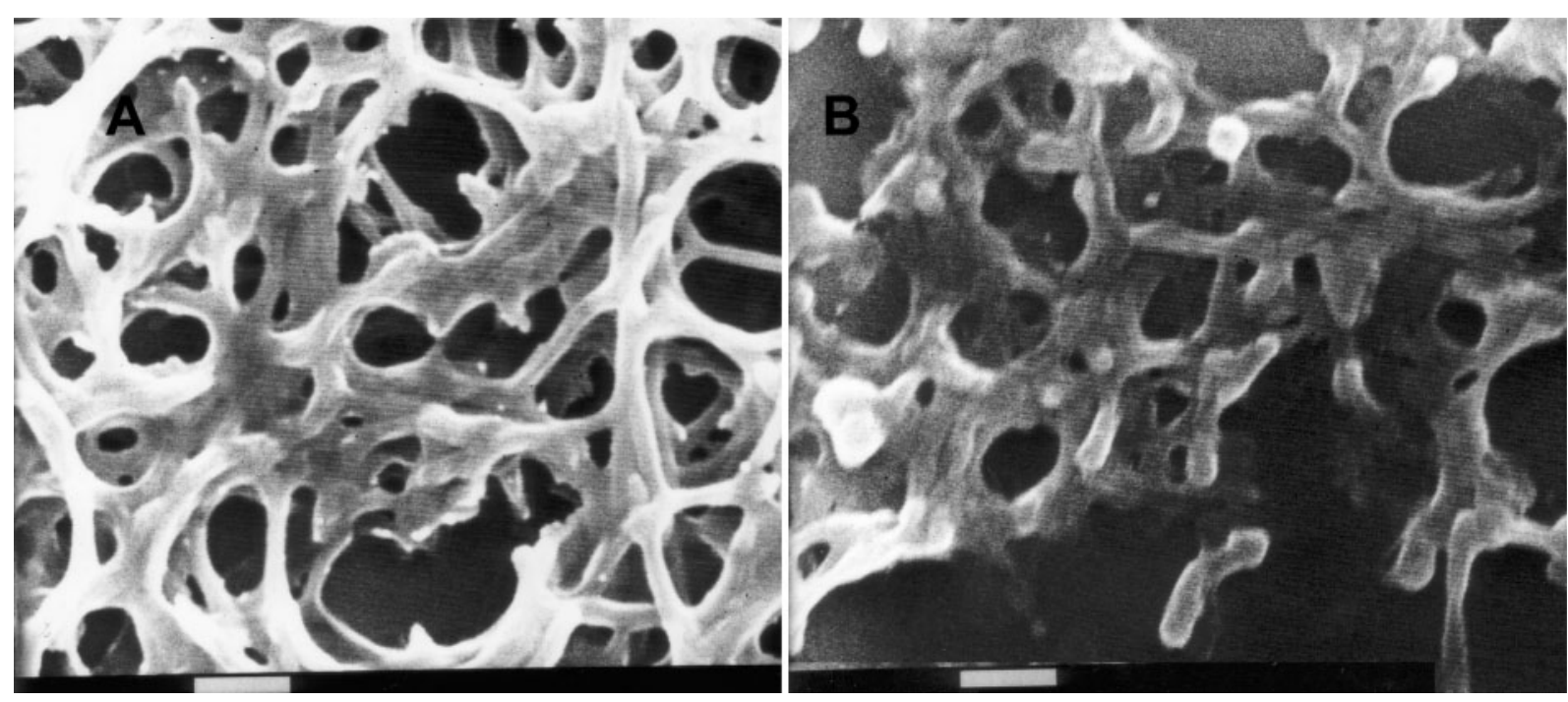

Fig. 4. SEM of aggregates formed by C. jejuni $11168 \mathrm{H}$ mutants: $\mathrm{A}, k p s M ; \mathrm{B}$, maf5. Bars, $1 \mu \mathrm{m}$.

The aggregated forms of biofilms of isogenic capsular polysaccharide ( $k p s M$ ) and flagellar (maf5) mutants (Fig. 4) were identical to that of the wild-type, precluding the requirement for capsular polysaccharide or flagella in the aggregate biofilm formation. The kpsM mutant's attached and pellicular biofilms again resembled those of the wildtype (Fig. 5A, B). However, the maf5 mutant attached minimally to glass (Fig. 5C) and did not form a pellicle (Fig. 1B); cells from the surface of a standing maf5 culture were not connected by an EPM and appeared as planktonic bacteria (Fig. 5D).

To assess the effect of biofilm on resistance to environmental stress, cultures were grown in triplicate for 3 days under microaerobic conditions promoting aggregate formation (see Methods). Aggregates and planktonic bacteria were then separated and transferred to ambient temperature and atmospheric conditions. Viable counts from individual cultures were taken at 3 day intervals. As seen in Fig. 6, while all planktonic bacteria died by 12 days following transfer to ambient conditions, bacterial aggregates survived for up to 24 days. Whereas cell numbers in planktonic cultures fell from $\sim 10^{9}$ c.f.u. $\mathrm{ml}^{-1}$ to zero by day 12 , viable cells in aggregates fell from $\sim 10^{9}$ to $10^{2}$ c.f.u. $\mathrm{ml}^{-1}$ by day 21 and to zero by day 24 .

\section{DISCUSSION}

Given the public health significance of $C$. jejuni infection it is important to understand how the organism survives in the environment and enters the food chain. Bacteria in a biofilm are relatively resistant to changes in environmental conditions, to antimicrobial agents and to host immune responses (Hall-Stoodley et al., 2004). It is an attractive hypothesis, therefore, that $C$. jejuni cells form a biofilm to survive adverse conditions between animal hosts; yet although $C$. jejuni has been found to colonize biofilms of autochthonous bacteria (Buswell et al., 1998), monospecies biofilm formation by $C$. jejuni has not previously been demonstrated.

We present data to show that $C$. jejuni in monoculture can form three distinct biofilms. It can attach to a glass surface (Figs 1C, 3B and 5A), and attachment is perhaps considered a hallmark and necessary first step in the formation of a biofilm. C. jejuni can also form a pellicle at a liquid-gas interface when grown in stationary culture (Figs $1 \mathrm{~B}, 3 \mathrm{C}$ and 5B), which has a similar three-dimensional architecture to the attached biofilm, with bacteria connected via an EPM, although the EPM is not flattened. Notably, $C$. jejuni forms unattached aggregates in liquid culture. Aggregates of this type have sometimes been referred to as flocs, which are said to resemble biofilms (Hall-Stoodley et al., 2004). We have used the term aggregate biofilm to describe the unattached bacteria connected to each other via an EPM and resembling the biofilm attached to glass and the pellicle forming at the liquid-gas interface. We propose that all three forms are true biofilms.

C. jejuni aggregate biofilms have increased resistance to ambient environmental stress, bacteria in the aggregate biofilm decreasing in number but surviving for up to 24 days, in comparison to up to 12 days survival by planktonic bacteria (Fig. 6). Similar experiments comparing pellicular and attached biofilm survival with planktonic bacteria after transfer from microaerobic conditions to ambient temperature and aerobic conditions were also performed. However, pellicles fell from the surface after 10-12 days and were visible as aggregates at the base of the tubes. These aggregates were no longer, by definition, pellicles and experiments were discontinued. It was not possible, therefore, to determine whether the attached and pellicular forms of $C$. jejuni biofilm conferred the same 

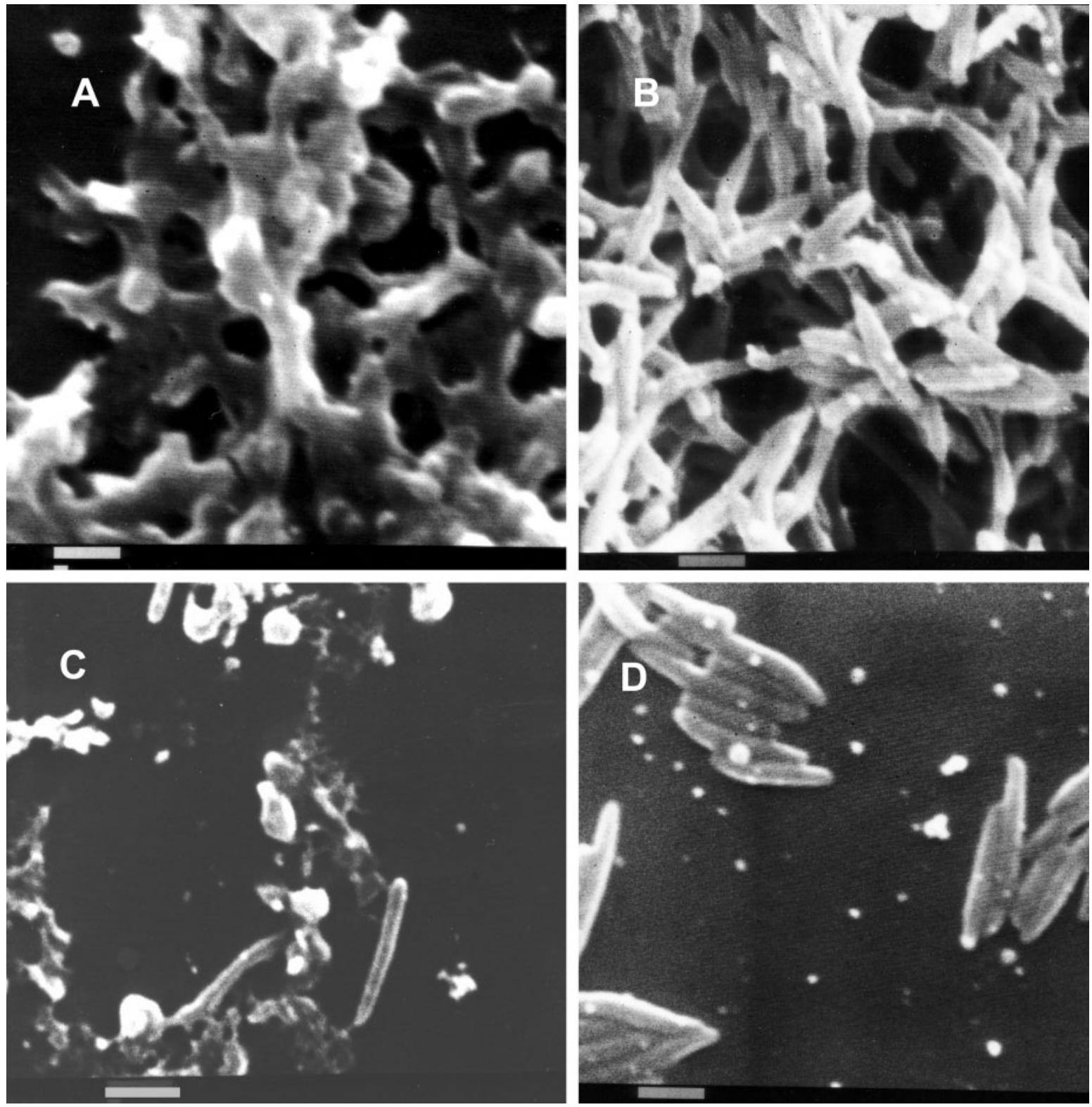

Fig. 5. SEM of C. jejuni $11168 \mathrm{H}$ mutants: $\mathrm{A}, k p s M$ attached; $\mathrm{B}, k p s M$ pellicle; $\mathrm{C}$, maf5 not attached; D, maf5 pellicle. Bars, $1 \mu \mathrm{m}$.

resistance to environmental stress. However, cells from the pellicle that were attached to the glass remained viable under atmospheric conditions for several weeks (unpublished data), suggesting that once a biofilm is formed (e.g. at $37^{\circ} \mathrm{C}$ ) this may provide a survival advantage to cells in the ambient environment. Due to stringent growth requirements $C$. jejuni cannot be grown at ambient temperature or atmosphere conditions; however we were able to demonstrate aggregate and pellicle formation for strain NCTC 11168 at $30^{\circ} \mathrm{C}$ under microaerophilic conditions (unpublished data). The relevance of biofilm formation for $C$. jejuni in its natural life cycle in the environment can only be speculated upon, as our studies demonstrating biofilm formation were undertaken at $37^{\circ} \mathrm{C}$ (or $30^{\circ} \mathrm{C}$ ). It may be that biofilm formation is possible within the host (e.g. avian, livestock or human), which subsequently confers a survival advantage to the micro-organism in the ambient environment (or a specific microenvironment) before transmission to the next host.

It is noteworthy that $C$. jejuni is strongly adherent to glass only at the liquid-gas interface, contiguous with the pellicle, in standing culture and does not attach to any surface we have tested with any shear force operating (flow or shaking). There may, however, be conditions in which attachment and subsequent biofilm formation occur that we have not 


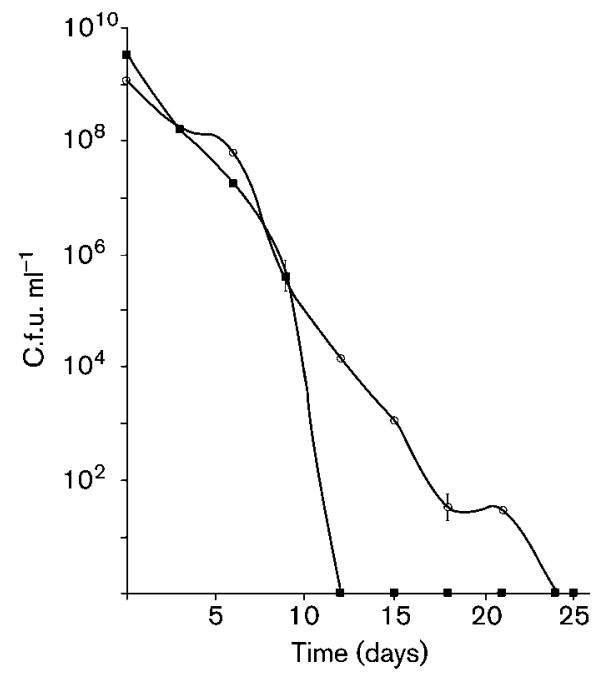

Fig. 6. Survival times of C. jejuni $11168 \mathrm{H}$ as aggregates $(\bigcirc)$ and as planktonic bacteria ( $\boldsymbol{\square})$. The error bars (some of which are smaller than the symbols) represent SD $(n=3)$.

determined. C. jejuni does, however, readily form the aggregate biofilm. It seems likely that aggregation of $C$. jejuni is initiated by non-specific forces such as hydrophobic interactions, as suggested by the relative hydrophobicity values of the wild-type and mutant strains (Table 1). The relative hydrophobicity was significantly reduced in aflagellate mutants, as previously observed by Misawa \& Blaser (2000). Hydrophobic interactions have also been proposed to allow C. jejuni cells to bind to INT 407 cell membranes (Moser \& Schroder, 1997). Whether or not subsequent development of the three forms of biofilm utilizes the same mechanisms and produces the same EPM remains to be determined.

Attachment by $C$. jejuni to a surface appears to be mediated by flagella, as the aflagellate fliS and maf5 mutants do not attach. Non-motile strains also failed to form pellicles, but in this case, attachment to a surface may not be a prerequisite for biofilm formation. A possible explanation may be that within the pellicle an oxygen gradient may form due to limited oxygen consumption; because C. jejuni are aerotactic (Marchant et al., 2002) this would disadvantage nonmotile strains. In addition, other genes may play a part in biofilm formation. Attachment is also reduced in the cj0688 mutant and it is noteworthy that the E. coli counterpart mutation (acp) also affected biofilm formation (Wolfe et al., 2003). In E. coli this acp gene encodes a phosphate transferase involved in the maintenance of the acetyl phosphate pool and also plays a crucial role in the cells' response to different environmental conditions such as nutrient concentration. This latter characteristic may be important in biofilm formation. By contrast, although tibA genes, which are responsible for glycosylation in enterotoxigenic E. coli, are important for biofilm formation (Sherlock et al., 2005), the $p g l$ genes that encode a general glycosylation pathway in
C. jejuni (Szymanski \& Wren, 2005) appear to have no role in biofilm formation in this species.

Laboratory-passaged strains $11168 \mathrm{H}$ and $81-176$, and a number of clinical isolates, form the aggregate biofilm, as well as strains with mutations in genes for capsular polysaccharide $(k p s M)$, flagella (maf5), glycoproteins $(p g l H)$, lipo-oligosaccharide (neuB1) and acetate kinase (cj0689). However, a number of wild-type strains, which possess flagella and are motile, do not form the aggregate biofilm; and strains mutant in another flagellar protein (FliS) and in a phosphate acetyltransferase (Cj0688) form a severely reduced aggregate biofilm. In addition, maf5 5 and fliS do not form a pellicle or attach to glass. Different molecular mechanisms, therefore, may control formation of the different forms of biofilm.

Given that aggregate biofilms were readily formed by the capsule-deficient mutant $k p s M$, the question remains what constitutes the EPM in C. jejuni biofilms. An alternative polysaccharide structure has been recently suggested that allows the Calcofluor white staining of $C$. jejuni cells (McLennan et al., 2005). Calcofluor white binds $\beta$-(1-4) and $\beta$-(1-3) sugar linkages and appears independent of the capsule. However, although the EPM of other bacterial biofilms is characteristically composed of polysaccharide, the genome sequence of $C$. jejuni does not show any obvious candidates for a biofilm polysaccharide other than the kps capsular polysaccharide gene cluster. It is possible, therefore, that the constitution of the biofilm may be modified capsular polysaccharides or poly-amino acid (Ornek et al., 2002), or it may be composed of extracellular DNA (Harris \& Mitchell, 1973; Whitchurch et al., 2002).

In conclusion, $C$. jejuni in monoculture can attach to surfaces and form a biofilm, and can form a pellicle at both $37^{\circ} \mathrm{C}$ and $30^{\circ} \mathrm{C}$. It also forms a biofilm growing unattached and this aggregate biofilm has increased resistance to environmental stress. This may be relevant to the survival of the organism in the environment and in the epidemiology of $C$. jejuni infection.

\section{ACKNOWLEDGEMENTS}

We thank Christine Szymanski and Bill Keevil for useful discussion on biofilm formation. We thank Maria McCrossan for assistance with electron microscopy studies. We acknowledge support from the Leverhulme Trust and the Biotechnology and Biological Sciences Research Council.

\section{REFERENCES}

Black, R. E., Levine, M. M., Clements, M. L., Hughes, T. P. \& Blaser, M. J. (1988). Experimental Campylobacter jejuni infection in humans. J Infect Dis 157, 472-479.

Buswell, C. M., Herlihy, Y. M., Lawrence, L. M., McGuiggan, J. T., Marsh, P. D., Keevil, C. W. \& Leach, S. A. (1998). Extended survival and persistence of Campylobacter spp. in water and aquatic biofilms 
and their detection by immunofluorescent-antibody and -rRNA staining. Appl Environ Microbiol 64, 733-741.

Costerton, J. W., Lewandowski, Z., Caldwell, D. E., Korber, D. R. \& Lappin-Scott, H. M. (1995). Microbial biofilms. Annu Rev Microbiol 49, 711-745.

CPLS (2000). Common gastrointestinal infections. Communicable Diseases Report Weekly England and Wales 10, 9-12.

Danese, P. N., Pratt, L. A. \& Kolter, R. (2000). Exopolysaccharide production is required for development of Escherichia coli $\mathrm{K}-12$ biofilm architecture. J Bacteriol 182, 3593-3596.

Domingue, G., Ellis, B., Dasgupta, M. \& Costerton, J. W. (1994). Testing antimicrobial susceptibilities of adherent bacteria by a method that incorporates guidelines of the National Committee for Clinical Laboratory Standards. J Clin Microbiol 32, 2564-2568.

Friedman, L. \& Kolter, R. (2004). Genes involved in matrix formation in Pseudomonas aeruginosa PA14 biofilms. Mol Microbiol 51, 675-690.

Golden, N. J. \& Acheson, D. W. (2002). Identification of motility and autoagglutination Campylobacter jejuni mutants by random transposon mutagenesis. Infect Immun 70, 1761-1771.

Hall-Stoodley, L., Costerton, J. W. \& Stoodley, P. (2004). Bacterial biofilms: from the natural environment to infectious diseases. Nat Rev Microbiol 2, 95-108.

Harris, R. H. \& Mitchell, R. (1973). The role of polymers in microbial aggregation. Annu Rev Microbiol 27, 27-50.

Huber, B., Riedel, K., Hentzer, M., Heydorn, A., Gotschlich, A., Givskov, M., Molin, S. \& Eberl, L. (2001). The cep quorum-sensing system of Burkholderia cepacia H111 controls biofilm formation and swarming motility. Microbiology 147, 2517-2528.

Karlyshev, A. V. \& Wren, B. W. (2005). Development and application of an insertional system for gene delivery and expression in Campylobacter jejuni. Appl Environ Microbiol 71, 4004-4013.

Karlyshev, A. V., Linton, D., Gregson, N. A., Lastovica, A. J. \& Wren, B. W. (2000). Genetic and biochemical evidence of a Campylobacter jejuni capsular polysaccharide that accounts for Penner serotype specificity. Mol Microbiol 35, 529-541.

Karlyshev, A. V., Linton, D., Gregson, N. A. \& Wren, B. W. (2002). A novel paralogous gene family involved in phase-variable flagellamediated motility in Campylobacter jejuni. Microbiology 148, 473-480.

Keevil, C. W. (2003). Rapid detection of biofilms and adherent pathogens using scanning confocal laser microscopy and episcopic differential interference contrast microscopy. Water Sci Technol 47, 105-116.

Kirov, S. M., Castrisios, M. \& Shaw, J. G. (2004). Aeromonas flagella (polar and lateral) are enterocyte adhesins that contribute to biofilm formation on surfaces. Infect Immun 72, 1939-1945.

Linton, D., Karlyshev, A. V., Hitchen, P. G., Morris, H. R., Dell, A., Gregson, N. A. \& Wren, B. W. (2000). Multiple $N$-acetyl neuraminic acid synthetase $(n e u B)$ genes in Campylobacter jejuni: identification and characterization of the gene involved in sialylation of lipooligosaccharide. Mol Microbiol 35, 1120-1134.

Linton, D., Allan, E., Karlyshev, A. V., Cronshaw, A. D. \& Wren, B. W. (2002). Identification of $\mathrm{N}$-acetylgalactosamine-containing glycoproteins PEB3 and CgpA in Campylobacter jejuni. Mol Microbiol 43, 497-508.

Loo, C. Y., Corliss, D. A. \& Ganeshkumar, N. (2000). Streptococcus gordonii biofilm formation: identification of genes that code for biofilm phenotypes. J Bacteriol 182, 1374-1382.

Mack, D., Rohde, H., Dobinsky, S., Riedewald, J., Nedelmann, M., Knobloch, J. K., Elsner, H. A. \& Feucht, H. H. (2000). Identification of three essential regulatory gene loci governing expression of Staphylococcus epidermidis polysaccharide intercellular adhesin and biofilm formation. Infect Immun 68, 3799-3807.

Marchant, J., Wren, B. \& Ketley, J. (2002). Exploiting genome sequence: predictions for mechanisms of Campylobacter chemotaxis. Trends Microbiol 10, 155-159.

McLennan, M., Ringoir, D., Jarrell, H. C. Szymanski, C. \& Gaynor, E. (2005). Characterisation of Campylobacter jejuni surface moiety that cross-reacts with calcofluor white: implications for surface carbohydrates, stress response, and pathogenesis. Abstracts of CHRO Meeting F32, 87.

Misawa, N. \& Blaser, M. J. (2000). Detection and characterization of autoagglutination activity by Campylobacter jejuni. Infect Immun $\mathbf{6 8}$, 6168-6175.

Moser, I. \& Schroder, W. (1997). Hydrophobic characterization of thermophilic Campylobacter species and adhesion to INT 407 cell membranes and fibronectin. Microb Pathog 22, 155-164.

Nesper, J., Lauriano, C. M., Klose, K. E., Kapfhammer, D., Kraiss, A. \& Reidl, J. (2001). Characterization of Vibrio cholerae O1 El tor galU and galE mutants: influence on lipopolysaccharide structure, colonization, and biofilm formation. Infect Immun 69, 435-445.

Nichols, W. W. (1991). Biofilms, antibiotics and penetration. Rev Med Microbiol 2, 177-181.

Ornek, D., Jayaraman, A., Syrett, B. C., Hsu, C. H., Mansfeld, F. B. \& Wood, T. K. (2002). Pitting corrosion inhibition of aluminum 2024 by Bacillus biofilms secreting polyaspartate or gamma-polyglutamate. Appl Microbiol Biotechnol 58, 651-657.

Parkhill, J., Wren, B. W., Mungall, K. \& 18 other authors (2000). The genome sequence of the food-borne pathogen Campylobacter jejuni reveals hypervariable sequences. Nature 403, 665-668.

Pearson, A. D., Greenwood, M. H., Feltham, R. K., Healing, T. D., Donaldson, J., Jones, D. M. \& Colwell, R. R. (1996). Microbial ecology of Campylobacter jejuni in a United Kingdom chicken supply chain: intermittent common source, vertical transmission, and amplification by flock propagation. Appl Environ Microbiol 62, 4614-4620.

Salloway, S., Mermel, L. A., Seamans, M., Aspinall, G. O., Nam Shin, J. E., Kurjanczyk, L. A. \& Penner, J. L. (1996). Miller-Fisher syndrome associated with Campylobacter jejuni bearing lipopolysaccharide molecules that mimic human ganglioside GD3. Infect Immun 64, 2945-2949.

Sherlock, O., Vejborg, R. M. \& Klemm, P. (2005). The TibA adhesin/ invasin from enterotoxigenic Escherichia coli is self recognizing and induces bacterial aggregation and biofilm formation. Infect Immun 73, 1954-1963.

Solano, C., Garcia, B., Valle, J., Berasain, C., Ghigo, J. M., Gamazo, C. \& Lasa, I. (2002). Genetic analysis of Salmonella enteritidis biofilm formation: critical role of cellulose. Mol Microbiol 43, 793-808.

Somers, E. B., Schoeni, J. L. \& Wong, A. C. (1994). Effect of trisodium phosphate on biofilm and planktonic cells of Campylobacter jejuni, Escherichia coli O157:H7, Listeria monocytogenes and Salmonella typhimurium. Int J Food Microbiol 22, 269-276.

Szymanski, C. M. \& Wren, B. W. (2005). Protein glycosylation in bacterial mucosal pathogens. Nat Rev Microbiol 3, 225-237.

Trachoo, N., Frank, J. F. \& Stern, N. J. (2002). Survival of Campylobacter jejuni in biofilms isolated from chicken houses. J Food Prot 65, 1110-1116.

van Vliet, A. H., Wooldridge, K. G. \& Ketley, J. M. (1998). Ironresponsive gene regulation in a Campylobacter jejuni fur mutant. J Bacteriol 180, 5291-5298. 
Watnick, P. I., Lauriano, C. M., Klose, K. E., Croal, L. \& Kolter, R. (2001). The absence of a flagellum leads to altered colony morphology, biofilm development and virulence in Vibrio cholerae O139. Mol Microbiol 39, 223-235.

Whitchurch, C. B., Tolker-Nielsen, T., Ragas, P. C. \& Mattick, J. S. (2002). Extracellular DNA required for bacterial biofilm formation. Science 295, 1487.

Whiteley, M., Ott, J. R., Weaver, E. A. \& McLean, R. J. (2001). Effects of community composition and growth rate on aquifer biofilm bacteria and their susceptibility to betadine disinfection. Environ Microbiol 3, 43-52.
Wolfe, A. J., Chang, D. E., Walker, J. D. \& 10 other authors (2003). Evidence that acetyl phosphate functions as a global signal during biofilm development. Mol Microbiol 48, 977-988.

Yuki, N. (1997). Molecular mimicry between gangliosides and lipopolysaccharides of Campylobacter jejuni isolated from patients with Guillain-Barre syndrome and Miller Fisher syndrome. J Infect Dis 176, Suppl 2, S150-S153.

Zimmer, M., Barnhart, H., Idris, U. \& Lee, M. D. (2003). Detection of Campylobacter jejuni strains in the water lines of a commercial broiler house and their relationship to the strains that colonized the chickens. Avian Dis 47, 101-107. 